\title{
Optimum Bandgap and Supply Voltage in Tunnel FETs
}

\author{
Qin Zhang, Member, IEEE, Yeqing Lu, Curt A. Richter, Senior Member, IEEE, \\ Debdeep Jena, Member, IEEE, and Alan Seabaugh, Fellow, IEEE
}

\begin{abstract}
A physics-based analytic model of the oN- and OFF-currents in a homojunction tunnel field-effect transistor (TFET) is used to understand the relationship between bandgap, gate length, ON-current, OFF-current, ON/OFF current ratio, and supply voltage to meet minimum energy requirements. The model, which applies to direct-bandgap semiconductors, is validated against numerical simulations to show that it captures the trends of more comprehensive simulations. The analytic model is then used to compare alternative channel materials for TFETs. Gate-all-around InAs nanowire and graphene nanoribbon TFETs are used as design examples at gate lengths of 10 and $15 \mathrm{~nm}$ and for an ON/OFF current specification of $10^{5}$. The results suggest that TFETs based on 2-D materials can be more energy efficient than semiconductor nanowire TFETs and conventional metal-oxide-semiconductor field-effect transistors for low-power logic.
\end{abstract}

Index Terms-Analytic model, graphene nanoribbon (GNR), nanowire (NW), tunnel field-effect transistor (TFET).

\section{INTRODUCTION}

$\mathbf{T}$ HE sub-60-mV/decade subthreshold swing of the tunnel field-effect transistor (TFET) allows reduction in supply voltage and power dissipation [1]-[3]. Projections based on III-V TFET atomistic simulations and experimentally calibrated models indicate that the TFET is a realistic candidate to complimentary metal-oxide-semiconductor at low supply voltages [4], [5].

In the development of TFETs, the quest for higher drive currents has led to the exploration of lower bandgap channels, for example, InGaAs [6]-[8] and InAs [9]-[11]. Vandenberghe et al. [12] showed, using a model based on the Wentzel-Kramers-Brillouin (WKB) approximation that there is an optimum bandgap and doping to maximize the ON current

Manuscript received July 11, 2013; revised April 23, 2014; accepted June 4, 2014. Date of current version July 21, 2014. This work was supported in part by the Semiconductor Research Corporation's Nanoelectronics Research Initiative through the Midwest Institute for Nanoelectronics Discovery and in part by the STARnet Center for Low Energy Systems Technology through the MARCO and DARPA. The review of this paper was arranged by Editor J. C. S. Woo.

Q. Zhang was with the University of Notre Dame, Notre Dame, IN 46556 USA, and also with the National Institute of Standards and Technology, Gaithersburg, MD 20899 USA. She is now with the University of Pisa, Pisa 56126, Italy (e-mail: qzhang1@nd.edu).

Y. Lu is with the University of Notre Dame, Notre Dame, IN 46556 USA (e-mail: ylu6@nd.edu).

C. A. Richter is with the National Institute of Standards and Technology, Gaithersburg, MD 20899 USA (e-mail: curt.richter@nist.gov).

D. Jena and A. Seabaugh are with the University of Notre Dame, Notre Dame, IN 46556 USA (e-mail: djena@nd.edu; seabaugh.1@nd.edu).

Color versions of one or more of the figures in this paper are available online at http://ieeexplore.ieee.org.

Digital Object Identifier 10.1109/TED.2014.2330805 at a particular supply voltage and without consideration of the OFF current. Khayer and Lake [13] provided an analytic approach for understanding the effect of channel material on both the ON and OFF currents in the TFET. In their assessment, the OFF-state leakage is considered to be independent of the gate length and given by interband tunneling from channel to drain at a supply voltage of $1 \mathrm{~V}$. Subsequent analysis by Luisier et al. [14] and Sylvia et al. [15] for 5-nm gate length transistors showed that direct source-to-drain tunneling dominates the OFF-state leakage and thus limits the ON/OFF current ratio.

Recently, Kam et al. [3] employed an analytic approach to evaluate the transfer characteristic of steep transistors to achieve a minimum energy-delay performance. Their analysis expanded the tradeoff space for optimizing TFETs to include subthreshold swing, ON-current, ON/OFF current ratio, activity factor, and logic depth, and showed that these must be balanced for steep devices to operate with lower energy and shorter delay than the metal-oxide-semiconductor field-effect transistor (MOSFET). The input for the analytic approach of Kam was the transfer characteristic of the steep transistor. Still lacking until now is a general approach that allows the tradeoffs to be explored between bandgap, gate length, ON-current, OFF-current, ON/OFF current ratio, and supply voltage to meet minimum energy requirements. While this can be done numerically and should be for a particular material system and device geometry, this is time consuming and does not yield physical insights across materials systems.

\section{Analytic Model And Validation}

To be shown is a simple analytic model of the ON and OFF currents for a homojunction direct-bandgap TFET that captures the essential physics of the TFET. This analysis applies to short channel transistors where direct source-to-drain tunneling dominates the OFF-state leakage and extends the results of [16] to $10 \mathrm{~nm}$ gate lengths and into 2-D materials [17], [18]. Our model captures the essential relationships among bandgap, gate length, and supply voltage in TFETs with excellent electrostatics such as in gate-all-around nanowire geometries [19] or in 2-D channel materials [18], [30]. The source/drain degeneracy is fixed for simplicity, but its optimization can be found in [12]. Trap-assisted tunneling, which has been observed experimentally [6], is neglected in the model, but could be added when this limitation in TFETs is better understood.

The schematic band diagrams for a generic $n$-channel homojunction $p-i-n$ TFET in the ON and OFF states are shown 


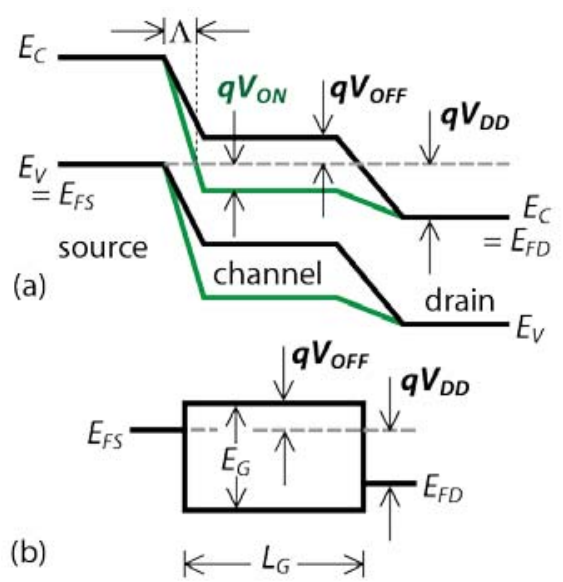

Fig. 1. (a) Schematic band diagram of an n-channel TFET in the ON (green) and OFF state (black). (b) Simplified band diagram in the OFF-state, assuming a source-to-channel junction width of $\Lambda$, which is much less than the gate length, $L_{G}$. The conduction band, valence band, and energy bandgap are given by their usual symbols, $E_{C}, E_{V}$, and $E_{G}$.

in Fig. 1, where the Fermi level is aligned with the valence band maximum in the source, $E_{F S}$, and the conduction band minimum in the drain, $E_{F D}$. In the ON state, the conduction band edge in the channel is pulled below the Fermi level in the source (dashed line) by a positive gate bias. This enables electrons to tunnel through a narrow energy barrier of width $\Lambda$, at the source-channel junction. The energy window for sourceto-channel tunneling in the ON state is $q V_{\mathrm{ON}}$, where $q$ is the electron charge and $V_{\mathrm{ON}}$ is the turn-on voltage. The energy $q V_{\mathrm{OFF}}$ defines the energy barrier between the source Fermi level and the channel conduction band in the OFF state.

In the OFF state, the leakage is dominated by direct tunneling from source to drain with an energy window given by $q V_{\text {DD }}$ [Fig. 1(b)]. The total potential change in the channel in going from the ON to the OFF state depends on the gatesource voltage, $V_{G S}$, which ranges as high as the supply voltage, $V_{\mathrm{DD}}$. A gate control parameter $\alpha, 0.8-0.9$ after Khayer and Lake [13], is used to set a proportionality between the gate-source voltage and $V_{\mathrm{ON}}$ while the coupling between the gate-source voltage and $V_{\mathrm{OFF}}$ is taken to be unity, assuming negligible injected charge in the channel in the OFF-state: $V_{\mathrm{ON}} / \alpha+V_{\mathrm{OFF}}=V_{\mathrm{DD}}$.

For short channel TFETs, both ON- and OFF-state currents are interband tunneling currents and are calculated by considering 1-D ballistic transport for nanowires and nanoribbons [2] and an abrupt Fermi function

$$
I=\frac{q}{\pi \hbar} \int T(E) d E
$$

where $\hbar$ is the reduced Plank constant, and $T(E)$ is the 1-D tunneling transmission coefficient. Here the spin degeneracy is 2 and the valley degeneracy is 1 . The lowest conduction subband and the highest valence subband are assumed to connect via a single dominant imaginary band in the bandgap for tunneling, which has been shown to be true in direct semiconductors from full-band atomistic simulations [21]. So the transmission coefficient can be calculated using

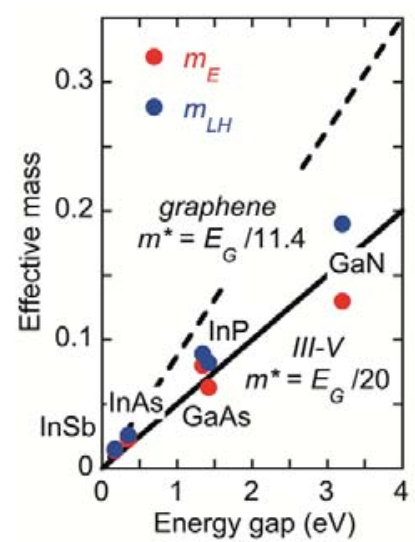

(a)

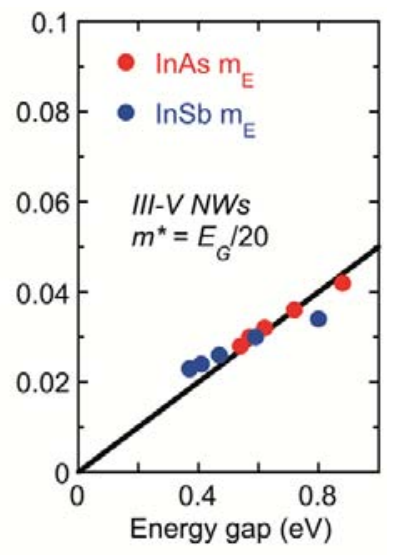

(b)
Fig. 2. (a) Electron effective mass (red dots) and light-hole effective mass (blue dots) versus energy bandgap for zinc-blende III-V semiconductors [23]. The electron effective mass, $m *$, is given by the electron effective mass, $m_{E}$ which is approximately equal to the light-hole effective mass, $m_{L H}$. (b) Electron effective mass versus bandgap for nanowires of InAs (red dots) and $\mathrm{InSb}$ (blue dots) with diameters in the range from 4 to $12 \mathrm{~nm}$ from Khayer and Lake [25].

Kane's two-band model [22]

$$
\begin{aligned}
T(E) & =\exp \left(-2 \int k d x\right) \\
& =\exp \left(-\frac{2 \sqrt{2 m_{E}}}{\hbar} \int \sqrt{E\left(1-E / E_{G}\right) d x}\right)
\end{aligned}
$$

where $\kappa$ is the magnitude of the imaginary wave vector and $m_{E}$ is the electron effective mass. In Kane's two-band model, the electron effective mass in the lowest conduction band is assumed to be equal to the light-hole effective mass in the highest valence band, which is reasonable for bulk zinc-blende III-V materials [23] with direct bandgaps [21]. It also applies for carbon-based materials that have a symmetric band structures.

The relationship between effective mass and bandgap is roughly linear for III-V semiconductors, as shown in Fig. 2(a) and as anticipated from two-band $k \cdot p$ theory [24]. For III-V semiconductors, the relationship is approximately $m_{E}=$ $E_{G} / 20$ where $E_{G}$ is given in eV. For nanowires (NWs), the energy bandgap increases with smaller NW diameter owing to quantum confinement. The calculated bandgaps and electron effective masses for InAs and InSb NWs are shown in Fig. 2(b) from Khayer and Lake [25], which uses a 3-D discretized eight-band $k \cdot p$ model. This model accounts for band nonparabolicity in the conduction band. The linear relation $m_{E}=E_{G} / 20$ is also still a good approximation for NWs as shown in Fig. 2(b). For graphene nanoribbons, the relationship between effective mass and bandgap is given by $m_{E}=$ $E_{G} / 11.4$ [26].

Using (2), the ON-state tunneling transmission coefficient, $T_{\mathrm{ON}}$, is calculated for electrons tunneling through a triangular energy barrier [27]

$$
T_{\mathrm{ON}}=\exp \left(-\frac{\pi \sqrt{2 m_{E}} E_{G}^{3 / 2}}{4 q \hbar \xi}\right)
$$




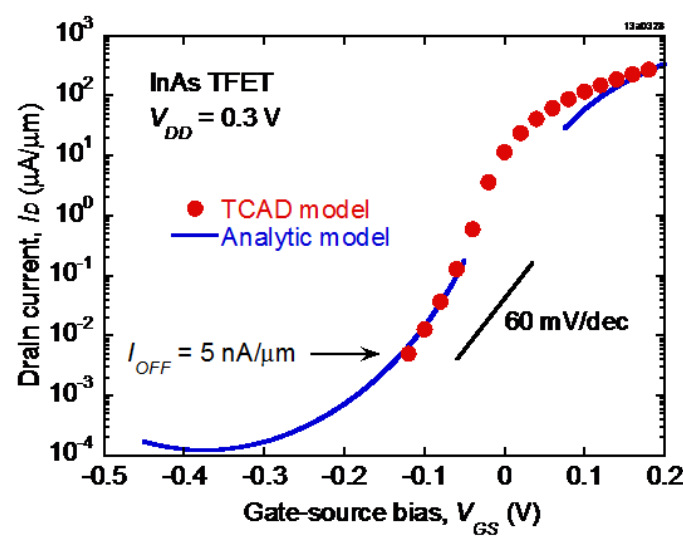

Fig. 3. Calculated transfer characteristics of an InAs GAA NW TFET (solid blue line) at a supply voltage of $0.3 \mathrm{~V}$ with $L_{G}=15 \mathrm{~nm}, t_{\mathrm{OX}}=0.6 \mathrm{~nm}$, $\epsilon_{\mathrm{OX}}=3.9 \epsilon_{O}, E_{G}=1.05 \mathrm{eV}$, and the nanowire diameter $d_{S}=3.5 \mathrm{~nm}$. The calculated results for the InAs GAA NW TFET from this paper is in fair agreement with TCAD simulation results (red dots) at $I_{\mathrm{OFF}}=5 \mathrm{nA} / \mu \mathrm{m}$.

with an average electric field in the tunneling direction given by $\xi=E_{G} /(q \Lambda)$. Here $\Lambda$ is related to the electrostatic length of the junction, $\lambda$, determined from a solution of Poisson's equation [28]. The electrostatic potential in the source-channel junction can be approximated as $\phi(x) \approx\left(E_{G} / q+V_{\mathrm{ON}}\right) \exp (-x / \lambda), V_{\mathrm{ON}}>0$ (see Fig. 1). It follows that at $x=0, \phi(0)=E_{G} / q+V_{\mathrm{ON}}$ and at $x=\Lambda, \phi(\Lambda)=\left(E_{G} / q+V_{\mathrm{ON}}\right) \exp (-\Lambda / \lambda)$ which equals $V_{\mathrm{ON}}$, then

$$
\Lambda=\lambda \ln \left(\frac{E_{G}+q V_{\mathrm{ON}}}{q V_{\mathrm{ON}}}\right) .
$$

The ON-state current is then written by substituting the energy-independent $\mathrm{ON}$-state tunneling transmission coefficient $T_{\mathrm{ON}}$ into (1) and completing the integration over the tunneling window

$$
I_{\mathrm{ON}}=\frac{q^{2}}{\pi \hbar} T_{\mathrm{ON}} V_{\mathrm{ON}}
$$

In the OFF-state, the tunneling transmission coefficient $T_{\mathrm{OFF}}$ is assumed to be approximated by electrons tunneling through a rectangular energy barrier with a length equal to the gate length. The flat channel profile [18], [19] is a good estimate for an Electrostatically well-designed TFET where the electrostatic scaling length is small relative to the gate length [29], [30]

$$
T_{\mathrm{OFF}}(E)=\exp \left(-\frac{2 \sqrt{2 m_{E} L_{G}}}{\hbar} \sqrt{E\left(1-E / E_{G}\right)}\right) .
$$

The OFF-state current is given by

$$
I_{\mathrm{OFF}}=\frac{q}{\pi \hbar} \int_{0}^{q V_{\mathrm{DD}}} T_{\mathrm{OFF}}\left(E+q V_{\mathrm{OFF}}\right) d E .
$$

To test this analytic model, the transfer characteristics of an InAs gate-all-around, $3.5 \mathrm{~nm}$ diameter, NW TFET are calculated and compared with Synopsys TCAD simulations [31] (see Fig. 3). In the TCAD model, drift-diffusion with Fermi statistics was used. Quantum confinement was

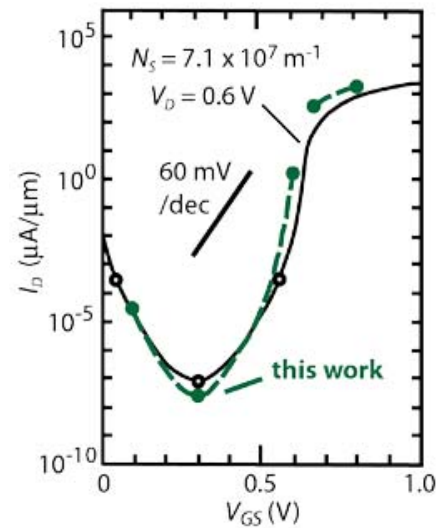

(a)

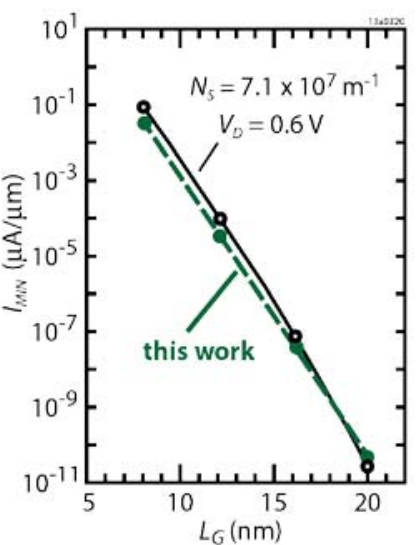

(b)
Fig. 4. (a) Calculated transfer characteristics of a GNR TFET (dashed line) at a supply voltage of $0.6 \mathrm{~V}$. The ribbon width $w=1.2 \mathrm{~nm}, E_{G}=1.22 \mathrm{eV}$, $L_{G}=16 \mathrm{~nm}, t_{\mathrm{OX}}=1 \mathrm{~nm}, \varepsilon_{\mathrm{OX}}=3.9 \varepsilon_{0}$. The calculated results for the GNR TFET in this paper is in fair agreement with NEGF [18] simulation results (solid line) where the source/drain doping is $7.1 \times 107 / m$. (b) Comparison of the minimum leakage current versus gate length from NEGF (solid line) [18] with the analytic model (dashed line) illustrating that it also captures the gate length dependence.

accounted for by adjusting the bandgap and density-ofstates effective masses obtained from tight-binding-simulated band structure. Tunneling was calculated using the Dynamic NonlocalPath tunneling model, where the tunneling effective mass was derived by fitting the imaginary band structure from tight binding. Even though the tunneling model in TCAD is based on the WKB approximation, the results were compared with NEGF quantum simulation and good agreement was achieved. Agreement was also shown in [28] with double-gate TFET simulations on $7 \mathrm{~nm}$ thin InGaAs channels.

In Fig. 3, the comparison of our analytic model with TCAD simulations is made at a gate length of $15 \mathrm{~nm}$, at a supply voltage of $0.3 \mathrm{~V}$ and for the same OFF current density, $5 \mathrm{nA} / \mu \mathrm{m}$. The analytic model is in good approximation to the numerical simulations in the bias ranges of interest. It is not exact, but provides a reasonable estimate for understanding tradeoffs and trends. Use of the model to explore tradeoffs at NW diameters below $3.5 \mathrm{~nm}$ should be proceeded by comparison and validation against reliable experimental results or simulations which comprehend quantization.

The transfer characteristics of a graphene nanoribbon TFET are also calculated analytically and compared with the NEGF method used by Chin et al. [18] [see Fig. 4(a)]. For this comparison, the gate length is $16 \mathrm{~nm}$, the supply voltage is $0.6 \mathrm{~V}$, and the ribbon width is $1.2 \mathrm{~nm}$. A source and drain doping concentration of $7.1 \times 10^{7} / \mathrm{m}$ is chosen to align the Fermi level with the valence and conduction edges, respectively, to minimize source depletion and to be consistent with the model energy band diagrams shown in Fig. 1. The analytic model captures the ambipolar transport in the TFET and provides a good representation of the ON- and OFF-state currents. The minimum currents predicted by the analytic model also capture the gate length dependence as shown in 


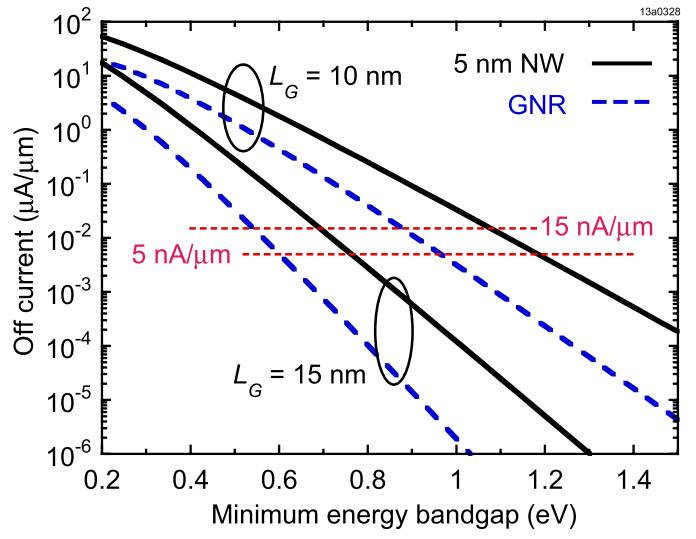

Fig. 5. OFF-state current versus the minimum energy bandgap, $E_{G}$, that is needed for NW TFETs (solid line) and GNR TFETs (dashed line) with gate lengths of 15 and $10 \mathrm{~nm}$. At a gate length, $L_{G}$, of $15 \mathrm{~nm}$, for an OFF-state current of $5 \mathrm{nA} / \mu \mathrm{m}$, the bandgap of the NW has to be at least $0.77 \mathrm{eV}$ and the bandgap of the GNR has to be at least $0.6 \mathrm{eV}$. As the gate length is scaled to $10 \mathrm{~nm}$, the minimum bandgaps of the NW and GNR rise to 1.19 and $0.93 \mathrm{eV}$, respectively, to meet this $I_{\mathrm{OFF}}=5 \mathrm{nA} / \mu$ m requirement.

Fig. 4(b) and are in agreement with the NEGF simulations of Chin et al. [18].

\section{OptimizATION OF BANDGAP AND SUPPly Voltage}

With an analytic model which captures the essential physics and trends, it becomes possible to explore the tradeoffs between bandgap and supply voltage. Following the circuitlevel energy-performance analysis of Kam et al. [3], the target ON/OFF current ratio is chosen to be $10^{5}$, the optimized ratio for TFETs. At this fixed optimized ON/OFF current ratio, the lower optimized supply voltage and lower effective subthreshold swing is an indicator of a more energy-efficient device. The electrostatic length, $\lambda$, is fixed to be 1.5 and $1.0 \mathrm{~nm}$ for the NW and GNR TFETs, respectively. All the currents are normalized to twice the NW diameter or twice the ribbon width.

The procedure for the optimization of bandgap and supply voltage proceeds in two steps. First, the minimum bandgap is determined to meet the OFF current requirement. This is determined from (7) which shows that the OFF current depends on both $V_{\mathrm{OFF}}$ and $V_{\mathrm{DD}}$; however, the minimum $V_{\mathrm{DD}}$ can be inferred from the band diagram shown in Fig. 1(b) by noting first that the relationship between the OFF-state voltage and supply voltage is $V_{\mathrm{OFF}}=\left(E_{G} / q-V_{\mathrm{DD}}\right) / 2$, or $V_{\mathrm{DD}}=E_{G} / q-$ $2 V_{\mathrm{OFF}}$. Next, note, from the earlier definition of $V_{\mathrm{ON}}, V_{\mathrm{ON}} / \alpha+$ $V_{\mathrm{OFF}}=V_{\mathrm{DD}}$, and the fact that $V_{\mathrm{ON}}$ is always positive, that $V_{\mathrm{OFF}}$ must always be less than $V_{\mathrm{DD}}$. Therefore, the minimum bandgap condition occurs when $V_{\mathrm{OFF}}=V_{\mathrm{DD}}=E_{G} /(3 q)$ and this then defines the minimum ofF current in (7).

The minimum OFF current versus bandgap is shown in Fig. 5 for a $5 \mathrm{~nm}$ diameter NW TFET and the GNR TFETs for two different gate lengths, 10 and $15 \mathrm{~nm}$. Two OFF current density requirements are considered, 5 and $15 \mathrm{nA} / \mu \mathrm{m}$ (with ON current of $1500 \mu \mathrm{A} / \mu \mathrm{m}$ ) corresponding to low-power (LP) and high-performance (HP) transistors, respectively. For this example, the minimum energy gap has to be at least $0.77 \mathrm{eV}$

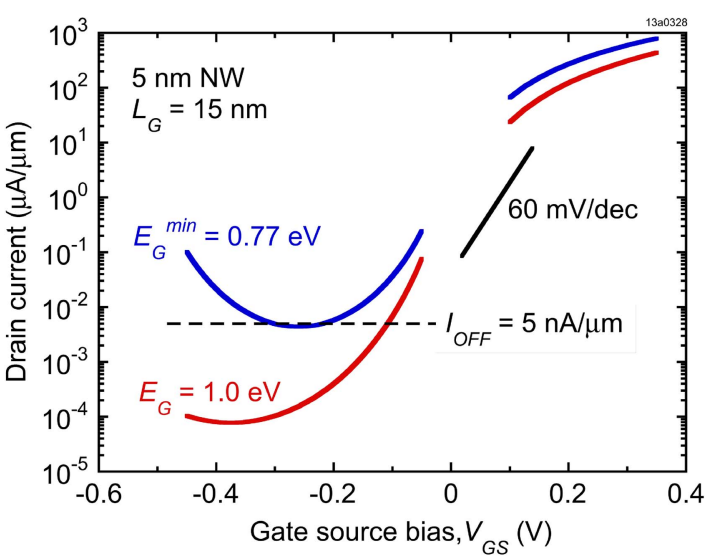

Fig. 6. Transfer characteristics of a $5 \mathrm{~nm}$ diameter InAs NW TFET with the minimum EG (dotted line) and a larger EG (solid line) for the required $I_{\mathrm{OFF}}=5 \mathrm{nA} / \mathrm{mm}$. It is shown that the TFET achieves a steeper subthreshold slope by increasing the bandgap from the minimum $E_{G}$ and reducing $V_{\mathrm{OFF}}$.

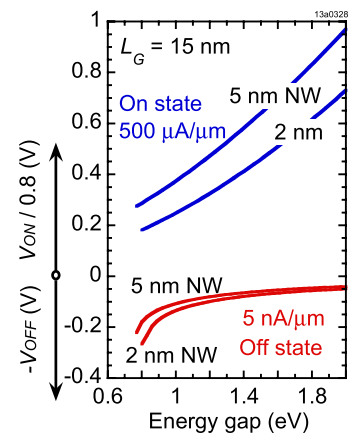

(a)

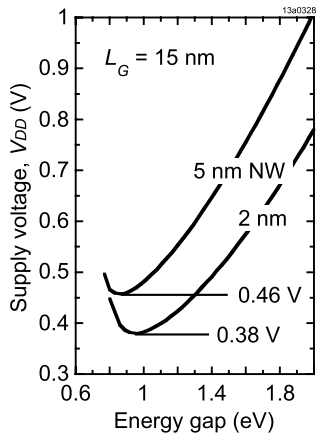

(b)
Fig. 7. Tradeoff space for TFETs: (a) For a given ON-OFF current ratio and gate length, constant current density plots show the relation between $V_{\mathrm{ON}}$, $V_{\mathrm{OFF}}$, and $E_{G}$ for the InAs NW TFET (diameter of 2 and $5 \mathrm{~nm}$ ). (b) Supply voltage versus bandgap showing an optimum bandgap to minimize the supply voltage.

to achieve an OFF current of $5 \mathrm{nA} / \mu \mathrm{m}$ for the $5 \mathrm{~nm}$ NW TFET with a gate length of $15 \mathrm{~nm}$.

The transfer characteristic of the $5 \mathrm{~nm}$ diameter InAs TFET is shown as the blue line in Fig. 6, confirming that the minimum OFF current of $5 \mathrm{nA} / \mu \mathrm{m}$ is obtained at $V_{\mathrm{OFF}}=E_{G} / q / 3$. It is also shown that this TFET can be switched at a steeper subthreshold slope with a larger energy bandgap and a smaller $V_{\mathrm{OFF}}$ (red line) by increasing the bandgap. However, it comes at the expense of lower ON current.

The design space can be compactly summarized as shown in Fig. 7(a) and (b). In Fig. 7(a), constant current plots are made to show how $V_{\mathrm{ON}}$ and $V_{\mathrm{OFF}}$ from (5) and (7) depend on bandgap for an ON/OFF ratio of $10^{5}$. Since $V_{\mathrm{ON}} / \alpha+V_{\mathrm{OFF}}=$ $V_{\mathrm{DD}}$ and $\alpha=0.8$, the supply voltage can be readily calculated for any band gap. $V_{\mathrm{DD}}$ is plotted versus bandgap in Fig. 7(b) where it is clear that there is an optimum bandgap of the channel material for a particular gate length and oN/OFF current requirement.

Similar plots can be made for the GNR TFET and these are shown in Fig. 8 where in addition to showing two gate 


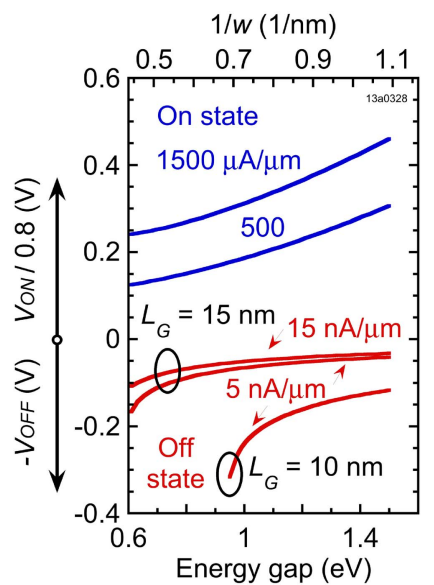

(a)

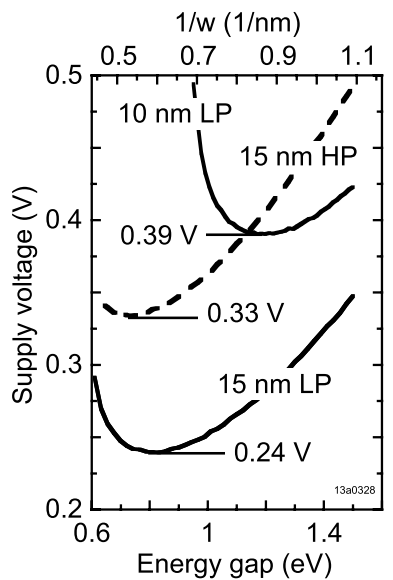

(b)
Fig. 8. (a) Similar plot as in Fig. 7(a) but with GNR TFETs. (b) For lowpower application, $I_{\mathrm{OFF}}=5 \mathrm{nA} / \mu \mathrm{m}$ and the $I_{\mathrm{ON}} / I_{\mathrm{OFF}}$ ratio of 105 , the minimum $V_{\mathrm{DD}}$ is 0.24 and $0.39 \mathrm{~V}$ for $L_{G}=15$ and $10 \mathrm{~nm}$, respectively. For high-performance application at $L_{G}=15 \mathrm{~nm}, I_{\mathrm{ON}}=1500 \mu \mathrm{A} / \mu \mathrm{m}$, and the $I_{\mathrm{ON}} / I_{\mathrm{OFF}}$ ratio of 105 , the minimum $V_{\mathrm{DD}}$ is $0.33 \mathrm{~V}$.

lengths the comparison is made for the two different OFF current requirements. Comparing Figs. 7(b) and 8(b), it is seen that the GNR TFETs have lower optimized supply voltage than the NW TFETs, which is due to the larger effective mass of graphene nanoribbons and the smaller scaling length for 2-D transistors [3]. In Fig. 8(b), it is shown that the optimized supply voltage increases with shorter gate length because bandgap must increase to meet the OFF-current requirement. Similarly, HP devices can meet the ON/OFF ratio specification with smaller bandgaps and the optimum supply voltage can take on a lower value for the LP technology.

\section{CONCLUSION}

A physics-based analytic model of advanced TFETs was formulated, compared with more sophisticated models, and then used to show how bandgap, supply voltage, and gatelength tradeoff to meet a given ON/OFF ratio. Both InAs NW and GNR TFET geometries were used to illustrate the model.

\section{REFERENCES}

[1] A. C. Seabaugh and Q. Zhang, "Low-voltage tunnel transistors for beyond CMOS logic," Proc. IEEE, vol. 98, no. 12, pp. 2095-2110, Dec. 2010.

[2] A. M. Ionescu and H. Riel, "Tunnel field-effect transistors as energyefficient electronic switches," Nature, vol. 479, no. 7373, pp. 329-337, Nov. 2011.

[3] H. Kam, T.-J. K. Liu, and E. Alon, "Design requirements for steeply switching logic devices," IEEE Trans. Electron Devices, vol. 59, no. 2, pp. 326-334, Feb. 2012.

[4] U. E. Avci, R. Rios, K. Kuhn, and I. A. Young, "Comparison of performance, switching energy and process variations for the TFET and MOSFET in logic," in Proc. Symp. VLSI Technol., Jun. 2011, pp. 124-125.

[5] U. E. Avci, S. Hasan, D. E. Nikonov, R. Rios, and I. A. Young, "Understanding the feasibility of scaled III-V TFET for logic by bridging atomistic simulations and experimental results," in Proc. Symp. VLSI Technol., Jun. 2012, pp. 183-184.
[6] S. Mookerjea et al., "Experimental demonstration of 100nm channel length In0.53Ga0.47As-based vertical inter-band tunnel field effect transistors (TFETs) for ultra low-power logic and SRAM applications," in Proc. Int. Electron Devices Meeting (IEDM), Dec. 2009, pp. 1-4.

[7] H. Zhao, Y. Chen, Y. Wang, F. Zhou, F. Xue, and J. Lee, "InGaAs tunneling field-effect-transistors with atomic-layer-deposited gate oxides," IEEE Trans. Electron Device, vol. 58, no. 9, pp. 2990-2995, Sep. 2011.

[8] G. Dewey et al., "Fabrication, characterization, and physics of III-V heterojunction tunneling field effect transistors (H-TFET) for steep sub-threshold swing," in Proc. Int. Electron Devices Meeting (IEDM), Dec. 2011, pp. 33.6.1-33.6.4

[9] K. E. Moselund, H. Schmid, C. Bessire, M. T. Bjork, H. Ghoneim, and H. Riel, "InAs-Si nanowire heterojunction tunnel FETs," IEEE Electron Device Lett., vol. 33, no. 10, pp. 1453-1455, Oct. 2012.

[10] G. Zhou et al., "Novel gate-recessed vertical InAs/GaSb TFETs with record high ION of $180 \mu \mathrm{A} / \mu \mathrm{m}$ at VDS $=0.5 \mathrm{~V}$," in Proc. Int. Electron Devices Meeting (IEDM), Dec. 2012, pp. 32.6.1-32.6.4.

[11] A. W. Dey et al., "High-current $\mathrm{GaSb} / \mathrm{InAs}(\mathrm{Sb})$ nanowire tunnel field-effect transistors," IEEE Electron Devices Lett., vol. 34, no. 2, pp. 211-213, Feb. 2013.

[12] W. G. Vandenberghe et al., "A model determining optimal doping concentration and material's band gap of tunnel field-effect transistors," Appl. Phys. Lett., vol. 100, no. 19, p. 193509, May 2012.

[13] M. A. Khayer and R. K. Lake, "Drive currents and leakage currents in $\mathrm{InSb}$ and InAs nanowire and carbon nanotube band-to-band tunneling FETs," IEEE Electron Devices Lett., vol. 30, no. 12, pp. 1257-1259, Dec. 2009.

[14] M. Luisier, M. Lundstrom, D. A. Antoniadis, and J. Bokor, "Ultimate device scaling: Intrinsic performance comparisons of carbonbased, InGaAs, and $\mathrm{Si}$ field-effect transistors for $5 \mathrm{~nm}$ gate length," in Proc. Int. Electron Devices Meeting (IEDM), Dec. 2011, pp. 11.2.1-11.2.4.

[15] S. S. Sylvia, H.-H. Park, M. A. Khayer, K. Alam, G. Klimeck, and R. K. Lake, "Material selection for minimizing direct tunneling in nanowire transistors," IEEE Trans. Electron Devices, vol. 59, no. 8, pp. 2064-2069, Aug. 2012.

[16] Q. Zhang, Y. Lu, C. A. Curt, D. Jena, and A. Seabaugh, "Analytic determination of the optimum bandgap for a tunnel FET," in Proc. Device Res. Conf., 2012, pp. 1-2.

[17] Q. Zhang, T. Fang, H. Xing, A. Seabaugh, and D. Jena, "Graphene nanoribbon tunnel transistors," IEEE Electron Device Lett., vol. 29, no. 12, pp. 1344-1346, Dec. 2008.

[18] S.-K. Chin, D. Seah, K.-T. Lam, G. S. Samudra, and G. Liang, "Device physics and characteristics of graphene nanoribbon tunneling FETs," IEEE Trans. Electron Devices, vol. 57, no. 11, pp. 3144-3152, Nov. 2010.

[19] M. Luisier and G. Klimeck, "Atomistic full-band design study of InAs band-to-band tunneling field-effect transistors," IEEE Electron Device Lett., vol. 30, no. 6, pp. 602-604, Jun. 2009.

[20] J. Knoch and J. Appenzeller, "Tunneling phenomena in carbon nanotube field-effect transistors," Phys. Status Solidi A, vol. 205, no. 4, pp. 679-694, Apr. 2008.

[21] M. Luisier and G. Klimeck, "Simulation of nanowire tunneling transistors: From the Wentzel-Kramers-Brillouin approximation to fullband phonon-assisted tunneling," J. Appl. Phys., vol. 107, no. 8, pp. 084507-1-084507-6, Apr. 2010.

[22] E. O. Kane, "Zener tunneling in semiconductors," J. Phys. Chem. Solids, vol. 12 , no. 2 , pp. 181-188, 1959.

[23] (2013, Jul.). New Semiconductor Materials. Characteristics and Properties [Online]. Available: http://www.ioffe.ru/SVA/NSM/

[24] P. Yu and M. Cardona, Fundamentals of Semiconductors: Physics and Material Properties. New York, NY, USA: Springer-Verlag, 2010.

[25] M. A. Khayer and R. K. Lake, "Performance of $n$-type InSb and InAs nanowire field-effect transistors," IEEE Trans. Electron Devices, vol. 55, no. 11, pp. 2939-2945, Nov. 2008.

[26] W. Vandenberghe, B. Soree, W. Magnus, and G. Groeseneken, "Zener tunneling in graphene based semiconductors-The k.p method," in Proc. Int. Conf. Electron Dyn. Semicond., OptoElectron. Nanostruct. vol. 193. 2009, pp. 1-4.

[27] E. O. Kane, "Theory of tunneling," J. Appl. Phys., vol. 32, no. 1, pp. 83-91, Jan. 1961.

[28] L. Liu, D. Mohata, and S. Datta, "Scaling length theory of double-gate interband tunnel field-effect transistors," IEEE Trans. Electron Devices, vol. 59, no. 4, pp. 902-908, Apr. 2012. 
[29] C. P. Auth and J. D. Plummer, "Scaling theory for cylindrical, fullydepleted, surrounding-gate MOSFET's," IEEE Electron Device Lett., vol. 18, no. 2, pp. 74-76, Feb. 1997.

[30] Q. Zhang, Y. Lu, H. G. Xing, S. J. Koester, and S. O. Koswatta, "Scalability of atomic-thin-body (ATB) transistors based on graphene nanoribbons," IEEE Electron Device Lett., vol. 31, no. 6, pp. 531-533, Jun. 2010.

[31] Sentaurus Device User Guide Version E, Synopsys Inc., Mountain View, CA, USA, 2010.

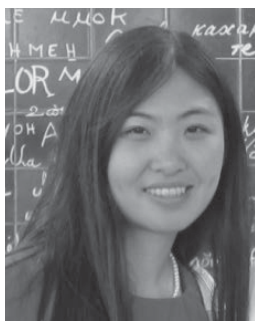

Qin Zhang (M'09) received the B.S. degree in electronic engineering from Tsinghua University, Beijing, China, in 2003, and the M.S. and Ph.D. degrees in electrical engineering from the University of Notre Dame, Notre Dame, IN, USA, in 2005 and 2009, respectively.

She was with the Department of Information Engineering, University of Pisa, Pisa, Italy, from 2013 to 2014. Her current research interests include tunneling devices and graphene electronics.

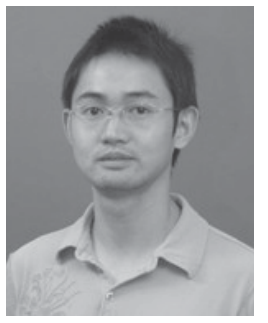

Yeqing Lu received the B.S. degree in physics from Zhejiang University, Hangzhou, China, in 2007, and the M.S. degree in electrical engineering from the University of Notre Dame, Notre Dame, IN, USA, where he is currently pursuing the Ph.D. degree.

$\mathrm{He}$ is currently an Application Engineer with Synopsys Inc., Mountain View, CA, USA. His current research interests include semiconductor device simulation.

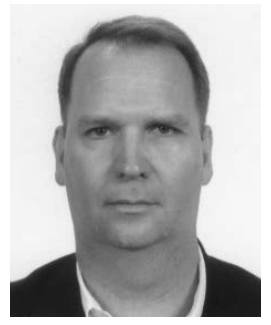

Curt A. Richter (SM'02) received the B.S. degree from the College of William and Mary, Williamsburg, VA, USA, in 1987, and the M.S., M.Phil., and $\mathrm{Ph} . \mathrm{D}$. degrees in applied physics from Yale University, New Haven, CT, USA, in 1990, 1991, and 1993, respectively.

He joined the National Institute of Standards and Technology, Gaithersburg, MD, USA, where he is a leader of the Nanoelectronics Group.

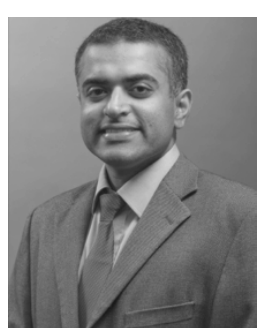

Debdeep Jena (M'99) received the B.Tech. degree in electrical engineering from the IIT Kanpur, Kanpur, India, in 1998, and the Ph.D. degree in electrical and computer engineering from the University of California at Santa Barbara, Santa Barbara, CA, USA, in 2003.

He joined the faculty of the Department of Electrical Engineering at the University of Notre Dame, Notre Dame, IN, USA, in 2003.

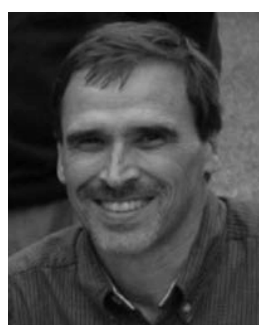

Alan Seabaugh (S'78-M'79-SM'92-F'03) received the $\mathrm{Ph} . \mathrm{D}$. degree in electrical engineering from the University of Virginia, Charlottesville, VA, USA in 1985.

$\mathrm{He}$ is a Professor of Electrical Engineering with the University of Notre Dame, Notre Dame, IN, USA, where he is also the Director of the SRC/DARPA STARnet Center for Low Energy Systems Technology. 